\title{
Are inflammatory and malnutrition markers associated with metabolic syndrome in patients with sarcoidosis?
}

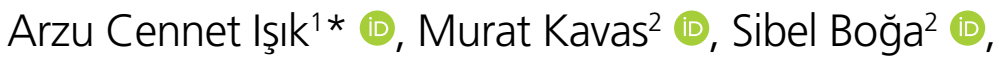 \\ Ali Karagöz ${ }^{3}$ (1), Gönenç Kocabay ${ }^{3}$ (D), Nesrin Şen ${ }^{4}$
}

\section{SUMMARY}

OBJECTIVE: The study aimed to investigate the use of Neutrophi//ymphocyte ratio, C-reactive protein/albumin ratio, controlling nutritional status, and prognostic nutritional index immune, inflammatory, and malnutrition markers Metabolic syndrome+ in sarcoidosis patients, as an early-stage marker.

METHOD: This is a single-center and cross-sectional study that determines the association of Metabolic syndrome in patients with sarcoidosis. Patients were evaluated based on the National Cholesterol Education Program's Adult Treatment Panel III criteria. Neutrophil/ lymphocyte ratio, C-reactive protein/albumin ratio, controlling nutritional status, and prognostic nutritional index values were simultaneously determined through blood test.

RESULTS: A total of 253 patients diagnosed with sarcoidosis were included in this study. Metabolic syndrome- was detected in $37.2 \%$ of patients. The prevalence was significantly higher in females $(p<0.001)$. Any degree of malnutrition assessed by controlling nutritional status had higher Metabolic syndrome ( $p=0.035$ ). The Neutrophil/lymphocyte ratio cutoff value was 2.24 , sensitivity was 70.53 , specificity was 60.13, and Area Under the Curve value was 0.663 for predicting Metabolic syndrome in sarcoidosis patients.

CONCLUSION: Neutrophil/lymphocyte ratio and controlling nutritional status are associated with the Metabolic syndrome+ in sarcoidosis patients. Thus, close monitoring of Neutrophi/lymphocyte ratio and controlling nutritional status increase in terms of Metabolic syndrome and immune malnutrition may be important in sarcoidosis patients.

KEYWORDS: Metabolic syndrome. Sarcoidosis. Neutrophils. Lymphocytes. Abdominal obesity. C-reactive protein. Albumin. Prognostic nutritional index.

\section{INTRODUCTION}

Sarcoidosis is a chronic systemic granulomatous disease that commonly affects the lungs. In the course of this disease, neurological findings, uveitis, blindness, end-stage pulmonary fibrosis, pulmonary hypertension, arrhythmia, cardiomyopathy, hypercalcemia, and renal failure may develop; approximately one-third of these side effects progresses as a chronic disease ${ }^{1}$.

Metabolic syndrome (MetS) is a heterogeneous disease that develops on the basis of insulin resistance and involves the combination of systemic disorders such as abdominal obesity,

\footnotetext{
'University of Health Sciences, Kartal Dr. Lütfi Kırdar City Hospital, Internal Medicine Department - Istanbul, Turkey.

¿2University of Health Sciences, Süreyyapașa Chest Diseases and Thoracic Surgery Training and Research Hospital, Department of Pulmonology Istanbul, Turkey.

${ }^{3}$ University of Health Sciences, Kartal Koșuyolu Training and Research Hospital, Department of Cardiology - Istanbul, Turkey.

${ }^{4}$ University of Health Sciences, Kartal Dr. Lütfi Kırdar City Hospital, Department of Rheumatology - Istanbul, Turkey.

*Corresponding author: arzukaracelik@gmail.com

Conflicts of interest: the authors declare there are no conflicts of interest. Funding: none.

Received on September 22, 2021. Accepted on September 22, 2021.
} 
glucose intolerance or diabetes mellitus, dyslipidemia, hypertension, and coronary artery disease (CAD) $)^{2}$. The neutrophil/ lymphocyte ratio (NLR) is a systemic inflammatory marker that can be easily measured and used in the prognosis of several chronic diseases.

In a recent study by Gülhan et al., the coexistence of MetS and insulin resistance was evaluated in patients with sarcoidosis and was found to be increased ${ }^{3}$. Due to Met $S$ components, the risk of early atherosclerosis and the presence of abdominal obesity are particularly important in terms of cardiovascular complications. Our study investigated the predictive value of NLR in predicting the incidence of MetS and the presence of MetS in sarcoidosis patients.

\section{METHODS}

The study was designed as an observational, cross-sectional study. The patients who were consecutively admitted as outpatient to the pulmonary medicine department were enrolled. The study was approved by the Local Ethics Committee.

All 345 patients diagnosed with sarcoidosis were screened cross-sectionally to evaluate MetS association and NLR according to the National Cholesterol Education Program's Adult Treatment Panel III (NCEP-ATP III) criteria. Those who had received or were planning to receive steroid therapy within the past six months, pregnant women, emergency patients, terminal-stage malignancies, and those with active and suspected infectious diseases were excluded from the study. Finally, a total of 253 patients, 94 sarcoidosis with MetS patients and 159 sarcoidosis without MetS patients, were included in the study.

The presence of diabetes mellitus (DM), hypertension (HT), hyperlipidemia (HL), and cardiovascular disease (CVD) was questioned. Based on additional examinations and follow-ups, those who were diagnosed for the first time had DM, HT, HL, and CVD.

\section{Diagnosis of sarcoidosis}

Definite diagnosis of sarcoidosis was established through fiber optic bronchoscopy (FOB) in 21.3\% ( $\mathrm{n}=53$ ) of patients, endobronchial ultrasonography (EBUS) in $34.1 \%(n=85)$ of patients, mediastinoscopy in $38.2 \%$ ( $n=95$ ) of patients, lung biopsy (wedge) in $4 \%(n=10)$ of patients, skin biopsy in $2 \%(n=5)$ of patients, and lymph node excisional biopsy in the remaining $0.4 \%(\mathrm{n}=1)$ of patients.

\section{Anthropometric measurements}

Each patient underwent a physical examination and a detailed medical examination. Anthropometric measurements and blood pressure measurements were noted. Waist circumference was measured with a tape at the level midway between the lower rib margin and the iliac crest. Blood pressure was measured in the sitting position using a mercury sphygmomanometer with the patients' arm at the level of the heart after they had rested for $15 \mathrm{~min}$ in the outpatient clinic.

All patients were evaluated for MetS according to the NCEPATP III criteria ${ }^{4}$. The presence of at least three of the five factors defined by ATP III for Met $S$ was accepted as a diagnosis of MetS. European criteria (male $\geq 94 \mathrm{~cm}$; female $\geq 80 \mathrm{~cm}$ ) were used for waist circumference measurement.

\section{Biochemical analysis}

Blood samples were collected after $12 \mathrm{~h}$ of fasting. Fasting blood glucose, cholesterol, triglyceride, LDL cholesterol, HDL cholesterol, $\mathrm{HbA1C}$, insulin, angiotensin-converting enzyme (ACE), hemogram, albumin, and C-reactive protein (CRP) were analyzed. Neutrophil/lymphocyte and CAR (CRP/albumin ratio) were used for NLR. Prognostic nutritional index (PNI) was calculated as follows: $\mathrm{PNI}=10 \times$ serum albumin (g/ $\mathrm{dL})+0.005 \times$ total lymphocyte count. A value exceeding 2.5 for the homeostasis model assessment of insulin resistance (HOMA-IR) (fasting blood glucosexfasting insulin/22.5) ratio indicates insulin resistance.

\section{Statistical analysis}

Variables were investigated using analytical and visual methods (Shapiro-Wilk test and histogram) to determine whether or not they are normally distributed. Continuous variables were presented as mean $\pm \mathrm{SD}$; if the variables are non-normally distributed, they are presented as median and interquartile range (IQR) $25-75 \%$. Categorical variables were depicted as percentages and numbers. Group comparisons were tested using independent sample t-test or the Mann-Whitney U test, according to distribution of the numerical variables; the chi-square test or the Fisher's exact test was used for the categorical variables. The association between MetS (outcome variable: MetS with sarcoidosis presence) and the CAR, PNI, NLR, age, LDL, and HOMA-IR variables was evaluated using the univariable and multivariable logistic regression models. In addition, receiver operating characteristic (ROC) curve analysis was used to determine whether NLR had discriminative ability for MetS. The independent contribution of each variable to the variance of outcome was estimated. In this regard, the relative importance of each predictor in the model was estimated with a partial $2^{\prime}$ value for each predictor. In addition, correlation analysis was performed for PNI, CAR, HOMA-IR, ACE, and NLR. In all statistical analyses, $\mathrm{p}<0.05$ was considered statistically significant. R software version 4.00 (Vienna, Austria) was used for the statistical analysis. 


\section{RESULTS}

The study population comprised of 253 patients (190 female patients). There was no statistically significant difference between the groups in terms of age, LDL cholesterol, waist circumferences, lymphocyte, insulin, PNI, and ACE. The MetS+ patients had higher neutrophil, CRP, CAR, and NLR than the MetSpatients, other baseline characteristics were described in Table 1.

In sarcoidosis patients, the LDL cholesterol value was $134 \pm 33.7 \mathrm{mg} / \mathrm{dL}$, serum triglyceride value was 125 (96-186) $\mathrm{mg} / \mathrm{dL}$, HDL cholesterol value was $45 \pm 11.2 \mathrm{mg} / \mathrm{dL}$, fasting blood glucose value was $98(90-190) \mathrm{mg} / \mathrm{dL}$, HbA1c was 6\%, waist circumference was $96 \pm 11.9 \mathrm{~cm}$, lymphocyte value was $180010^{3} / \mu \mathrm{L}$ (1400-2300), neutrophil value was $390010^{3} / \mu \mathrm{L}$ (3100-5000), CRP was $4.2 \mathrm{mg} / \mathrm{dL}$ (3.2-7.0), albumin was 4.3 $\mathrm{g} / \mathrm{L}(4.1-4.5)$, and insulin was $11.4 \mathrm{mIU} / \mathrm{L}$ (8.1-17); $28.9 \%$ $(n=73)$ of patients had HT, $19 \%(n=48)$ had DM, and $49.4 \%$ $(n=125)$ had elevated triglyceride levels. Waist circumference was increased in $80.3 \%$ of women $(n=151)$ and was statistically significant compared with men $(\mathrm{p}<0.001)$. It was increased in $19.7 \%(n=12)$ of the male patients, and the median waist circumference was $96 \mathrm{~cm} ; 37.2 \%(\mathrm{n}=94)$ of patients had MetS. In terms of distribution, $89.3 \%(n=84)$ of women and $10.7 \%$ $(\mathrm{n}=10)$ of men had MetS $(\mathrm{p}<0.001)$. The median duration of disease in $\mathrm{Met} S+$ patients with sarcoidosis was found to be four years. Out of $94 \mathrm{MetS}+$ patients, $57(60.6 \%)$ had HT, and 25 (26.5\%) had DM. Out of the 159 MetS-patients, $16(10 \%)$ had HT, and 23 (14.4\%) had DM. Fasting blood glucose level was $111(97-125) \mathrm{mg} / \mathrm{dL}$ in MetS+ patients, whereas it was 94 (87-101) in MetS- patients. In MetS+ patients, the neutrophil value was $4700^{\prime} 10^{3} / \mu \mathrm{L}(3685-5900)$, CAR was 11.8 (8.01-20.6), NLR was 2.64 (2.09-3.48), and HOMA-IR was 6.73 (3.83-10.2), which were statistically significant $(\mathrm{p}<0.001)$.

Binary logistic regression analysis showed that NLR (OR 1.80 [1.21-2.68], $\mathrm{p}<0.001$ ), LDL (OR 1.49 [1.04-2.15], $\mathrm{p}=0.003$ ), and HOMA-IR (OR 1.37 [1.04-1.85], $\mathrm{p}=0.02$ ) were statistically significant compared with MetS-, while the other variables were not (Table 2).

The relative importance of each predictor in the model was presented in Figure 1A; the important variables such as NLR and HOMA-IR were used to predict the presence of MetS in

Table 1. Baseline demographic and clinical variables.

\begin{tabular}{|c|c|c|c|c|}
\hline & All $(n=253)$ & MetS- $(n=159)$ & MetS+ $(n=94)$ & p-value \\
\hline Age (years) & $48.6 \pm 11.4$ & $49.4 \pm 11$ & $47.1 \pm 12$ & 0.13 \\
\hline Gender (female \%) & $190(75.1)$ & $106(66.6)$ & $84(89.3)$ & $<0.001$ \\
\hline DM presence (\%) & $48(19)$ & $23(14.4)$ & $25(26.5)$ & 0.02 \\
\hline HT presence (\%) & $73(28.9)$ & $16(10)$ & $57(60.6)$ & $<0.001$ \\
\hline Glucose (mg/dL) & $98(90-109)$ & $94(87-101)$ & $111(97-125)$ & $<0.001$ \\
\hline $\mathrm{LDL}(\mathrm{mg} / \mathrm{dL})$ & $134 \pm 33.7$ & $133 \pm 34.7$ & $139 \pm 31.9$ & 0.17 \\
\hline $\mathrm{HDL}(\mathrm{mg} / \mathrm{dL})$ & $45 \pm 11.2$ & $49.9 \pm 12$ & $43.9 \pm 8.5$ & $<0.001$ \\
\hline Triglyceride (mg/dL) & $125(96-186)$ & $109(85.5-138)$ & $179(141-227)$ & $<0.001$ \\
\hline Waist circumference $(\mathrm{cm})$ & $96 \pm 11.9$ & $96.5 \pm 12$ & $97.5 \pm 11.7$ & 0.56 \\
\hline Albumin $(\mathrm{g} / \mathrm{L})$ & $4.3(4.1-4.5)$ & $4.3(4.13-4.50)$ & $4.3(4.10-4.50)$ & 0.46 \\
\hline Lymphocyte $\left(10^{3} / \mu \mathrm{L}\right)$ & 1800 (1400-2300) & 1800 (1375-2308) & 1800 (1400-2200) & 0.94 \\
\hline Neutrophil $\left(10^{3} / \mu \mathrm{L}\right)$ & 3900 (3100-5000) & $3600(2805-4400)$ & 4700 (3685-5900) & $<0.001$ \\
\hline CRP (mg/L) & $4.2(3.2-7.0)$ & $3.70(3.20-6.27)$ & $5.20(3.20-8.74)$ & 0.003 \\
\hline Insulin (mIU/L) & $11.4(8.1-17)$ & $10.9(8.3-14.5)$ & $13.4(8.30-18.8)$ & 0.053 \\
\hline CAR & $9.76(7.38-17.8)$ & $8.46(7.27-13.9)$ & $11.8(8.01-20.6)$ & $<0.001$ \\
\hline NLR & $2.25(1.67-3.15)$ & $2.10(1.56-2.75)$ & $2.64(2.09-3.48)$ & $<0.001$ \\
\hline PNI & $52.2(49-55.5)$ & $52.0(49-56)$ & $52.5(49-55.3)$ & 0.96 \\
\hline HOMA-IR & $5.12(3.69-7.86)$ & $4.76(3.51-6.42)$ & $6.73(3.83-10.2)$ & $<0.001$ \\
\hline $\mathrm{ACE}(\mathrm{U} / \mathrm{L})$ & $56.8(37.1-87.5)$ & $58.6(42.6-87.9)$ & $54.7(29.1-85.4)$ & 0.29 \\
\hline
\end{tabular}

MetS: Metabolic syndrome; DM: diabetes mellitus; HT: hypertension; LDL: low-density lipoprotein; HDL: high-density lipoprotein; CRP: C-reactive protein; CAR: C-reactive protein/albumin ratio; NLR: neutrophil/lymphocyte ratio; PNI: prognostic nutritional index; HOMA-IR: homeostatic model assessmentinsulin resistance; ACE: angiotensin-converting enzyme. All patients and MetS+ and MetS-. 
Table 2. Multivariable logistic regression for predict Metabolic syndrome presence in sarcoidosis.

\begin{tabular}{l|c|c|c} 
& $\begin{array}{c}\text { Multivariable } \\
\text { odds ratio }\end{array}$ & $95 \% \mathrm{Cl}$ & $\mathrm{p}$-value \\
\hline $\begin{array}{l}\text { CRP/albumin ratio } \\
\text { (from 7.38-17.76) }\end{array}$ & 1.14 & $0.99-1.32$ & 0.06 \\
\hline $\begin{array}{l}\text { Prognostic } \\
\text { nutritional index } \\
\text { (from 49-55) }\end{array}$ & 1.03 & $\begin{array}{c}0.67- \\
1.56\end{array}$ & 0.89 \\
\hline $\begin{array}{l}\text { Neutrophil/ } \\
\text { lymphocyte ratio } \\
\text { (from 1.66-3.14) }\end{array}$ & 1.80 & $1.21-2.68$ & 0.003 \\
\hline $\begin{array}{l}\text { LDL (from 112- } \\
\text { 154 mg/dL) }\end{array}$ & 1.49 & $1.04-2.15$ & 0.03 \\
\hline $\begin{array}{l}\text { HOMA-IR } \\
\text { (from 3.68-7.85) }\end{array}$ & 1.37 & $1.04-1.85$ & 0.02 \\
\hline $\begin{array}{l}\text { Age (from } \\
\text { 40-56 years) }\end{array}$ & 0.77 & $0.51-1.16$ & 0.22 \\
\hline
\end{tabular}

CRP: C-reactive protein; LDL: low-density lipoprotein; Cl: Confidence interval; CRP: C-reactive protein; LDL: low-density lipoprotein; HOMA-IR: homeostatic model assessment-insulin resistance. Bold values denote statistical significance at the $p<0.05$ level. the sarcoidosis patient. The partial effect plots show the fitted curve on the mean (probability) scale as log-odds (linear predictor) for NLR in Figure 1B.

The NLR cutoff value was 2.24 , sensitivity was 70.53 , specificity was 60.13 , and AUC was 0.663 in predicting MetS in sarcoidosis patients. Herein, a negative correlation existed between NLR and PNI in the correlation analysis [R $0.369(\mathrm{p}<0.001)]$. However, there was no correlation between HOMA-IR and NLR, PNI, and CAR.

\section{DISCUSSION}

This study showed that NLR was higher in sarcoidosis patients with MetS. Chuan-Chuan Liu et al. evaluated patients in six groups using anthropometric, biochemical, and hematological measurements in terms of MetS marker (NLR) in a study including 34,013 subjects. NLR was concluded to be a good predictor, and the risk increased as this ratio increased. NLR and increased values of NLR could be used as a prognostic marker for the development of MetS $S^{5}$. In another study, Kaya et al. investigated the relationship between NLR and CAD using syntax score (SS) in 649 patients with stable angina pectoris and CAD; they determined that NLR was a measurable
(A)

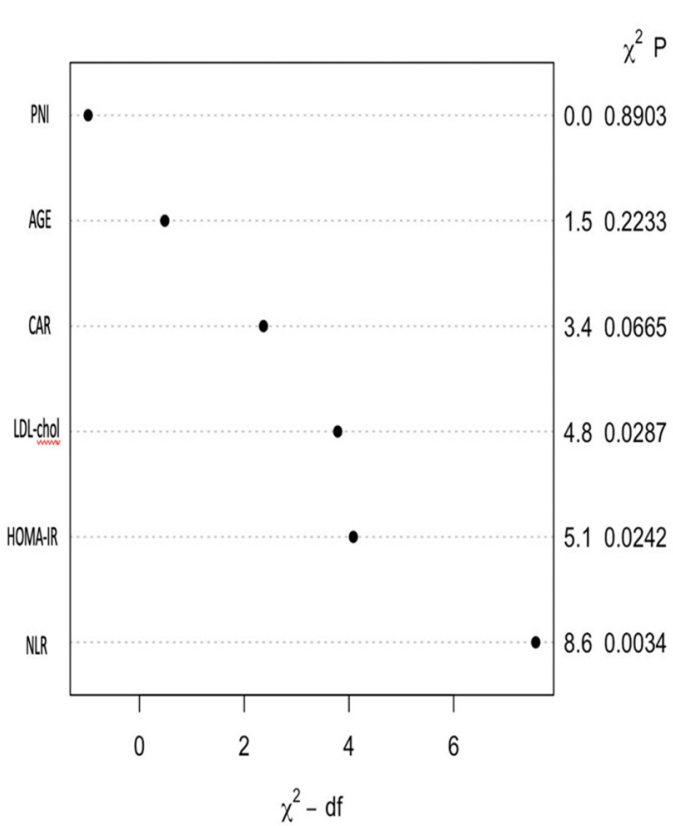

(B)

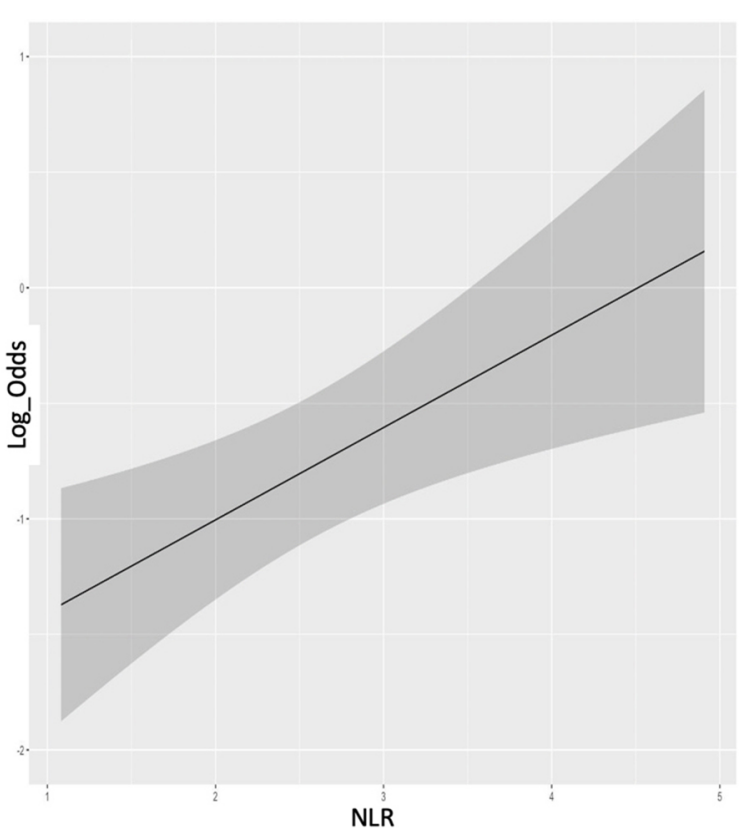

PNI: prognostic nutritional index; age: years; CAR: C-reactive protein/albumin ratio; LDL: low density lipoprotein; HOMA-IR: homeostatic model assessment-insulin resistance; NLR: neutrophil/lymphocyte ratio.

Figure 1. (A) Relative importance of each variable in the multivariable model for predict presence of metabolic syndrome in sarcoidosis. (B) Partial effect plot of neutrophil/lymphocyte ratio for predicting presence of metabolic syndrome+. 
systemic inflammatory marker. In multivariate analysis, NLR was associated with the presence and severity of $\mathrm{CAD}^{6}$.

In their studies including 1300 sarcoidosis patients, Güngör et al. investigated the use of NLR as a marker of inflammation in sarcoidosis. It was concluded that NLR could be used as an inflammation marker, and studies with large patient populations were needed for activity and staging in prognosis?

In the report of Balta et al., NLR value was suggested as an independent prognostic factor for $\mathrm{CAD}$, which may be affected by vascular disease-associated MetS, DM, HT, and hypercholesterolemia ${ }^{8}$. In their case-control study, Büyükkaya et al. divided MetS+ patients into three groups (based on their components). MetS+ patients had significantly higher NLR values compared with the control group, and it was observed that the NLR increased with increasing severity $(r=0.586, p<0.001)^{9}$. Similarly, as a result of our study, NLR was statistically significant as predicted by MetS+. In addition, in the study conducted by Bahadır et al, correlation analysis was performed by comparing metabolic and inflammatory markers between the groups, and it was concluded that NLR is not a good marker of inflammation, and leukocyte and hs-CRP values may be more useful biomarkers to indicate inflammation in nondiabetic patients with obesity and $\mathrm{Met} \mathrm{S}^{10}$.

In our study, $37.15 \% \mathrm{Met}$ + and 6.73 (3.83-10.2) HOMA-IR values were higher in women and were statistically significant $(\mathrm{p}<0.001)$. In the study conducted by Cozier et al., the relationship of obesity and weight gain with the incidence of sarcoidosis was evaluated in 59,000 US black women aged between 21 and 69 years; of these, the development of sarcoidosis was reported in 454 patients during a 16-year follow-up period (1995-2011). The incidence of sarcoidosis increased with increasing body mass index and weight gain ${ }^{11}$.

In the study conducted by Moon et al., patients with elevated CAR and DM were at higher risk of all-cause mortality compared with those without elevated CAR and $\mathrm{DM}^{12}$. Similarly, significant results were achieved with CAR and NLR values in predicting inflammation in MetS+ patients $(\mathrm{p}<0.001)$.
Gvozdenovic et al. conducted a case-control study with 184 patients and evaluated the effect of high body mass index (BMI) on patient-reported results in sarcoidosis patients and healthy individuals, and the highest risk (more than three times) was detected in obese women ${ }^{13}$. In this study, MetS+ was more common in women and was statistically significant $(\mathrm{p}<0.001)$. In the recently published review, the importance of inflammatory parameters was stated, but malnutrition was left out ${ }^{14}$. According to the results of our research, sarcoidosis patients may need to have their inflammation and malnutrition assessed.

\section{Limitations}

This study has some limitations. Being a single-center study and its observational nature is one of the limitations of our study. Our findings should be confirmed in prospective and largescale studies involving other inflammatory biomarkers to clarify the exact mechanistic role of NLR in sarcoidosis with MetS+.

\section{CONCLUSIONS}

In addition to classical parameters, NLR can be used in sarcoidosis patients to predict MetS+. The use of NLR, a strong inflammation marker, may be considered for the closer follow-up needed in patients with MetS+ sarcoidosis. Sarcoidosis patients should be followed up closely in terms of possible comorbidities through separate evaluation in terms of MetS components in their long-term follow-up.

\section{AUTHORS" CONTRIBUTIONS}

ACI: Conceptualization, Data curation, Formal Analysis, Investigation, Methodology, Writing-original draft. MK: Conceptualization, Data curation, Writing - original draft. SB: Data curation, Visualization. AK: Formal Analysis, Methodology, Visualization, Writing - original draft. GK: Formal Analysis, Investigation, Methodology. NŞ: Investigation, Methodology, Visualization.

\section{REFERENCES}

1. Iannuzzi MC, Fontana JR. Sarcoidosis: clinical presentation, immunopathogenesis, and therapeutics. JAMA. 2011;305(4):391-9. https://doi.org/10.1001/jama.2011.10

2. Arslan M, Atmaca A, Ayvaz G, Başkal N, Beyhan Z, Bolu E, et al. Metabolik Sendrom Kılavuzu [Internet]. Ankara: Turkish Journal of Endocrinology and Metabolism. 2009 [cited on Jul. 30, 2021]. Available from: http://temd.org.tr/admin/uploads/ tbl_yayinlar/metabolik_sendrom.pdf

3. Gülhan PY, Balbay EG, Erçelik M, Yıldız Ş, Yılmaz MA. Is sarcoidosis related to metabolic syndrome and insulin resistance? Aging Male. 2020;23(1):53-8. https://doi.org/10.1080/1368 5538.2019.1631272
4. Expert Panel on detection, evaluation, and treatment of high blood cholesterol in adults. Executive summary of the third report of The National Cholesterol Education Program (NCEP) expert panel on detection, evaluation, and treatment of high blood cholesterol in adults (Adult Treatment Panel III). JAMA. 2001;285(19):2486-97. https://doi.org/10.1001/jama.285.19.2486

5. Liu CC, Ko HJ, Liu WS, Hung CL, Hu KC, Yu LY, et al. Neutrophilto-lymphocyte ratio as a predictive marker of metabolic syndrome. Medicine (Baltimore). 2019;98(43):e17537. https:// doi.org/10.1097/MD.0000000000017537

6. Kaya A, Kurt M, Tanboga IH, Işık T, Günaydın ZY, Kaya Y, Topçu $\mathrm{S}$, Sevimli $\mathrm{S}$. Relation of neutrophil to lymphocyte ratio with 
the presence and severity of stable coronary artery disease. Clin Appl Thromb Hemost. 2014;20(5):473-7. https://doi. org/10.1177/1076029612473517

7. Gungor S, Akturk UA, Yalcinsoy M, Kocak ND, Goksenoglu NC, Altunbey SA, et al. What is the neutrophil/lymphocyte ratio in sarcoidosis? Bratisl Lek Listy. 2016;117(3):152-5. https://doi.org/10.4149/bll_2016_030

8. Balta S, Celik T, Mikhailidis DP, Ozturk C, Demirkol S, Aparci M, et al. The Relation between atherosclerosis and the neutrophillymphocyte ratio. Clin Appl Thromb Hemost. 2016;22(5):40511. https://doi.org/10.1177/1076029615569568

9. Buyukkaya E, Karakas MF, Karakas E, Akçay AB, Tanboga $I H$, Kurt $M$, et al. Correlation of neutrophil to lymphocyte ratio with the presence and severity of metabolic syndrome. Clin Appl Thromb Hemost. 2014;20(2):159-63. https://doi. org/10.1177/1076029612459675

10. Bahadır A, Baltacı D, Türker $Y$, Türker $Y$, lliev D, Öztürk $S$, et al. Is the neutrophil-to-lymphocyte ratio indicative of inflammatory state in patients with obesity and metabolic syndrome? Anatol J Cardiol. 2015;15(10):816-22. https:// doi.org/10.5152/akd.2014.5787

11. Cozier YC, Coogan PF, Govender P, Berman JS, Palmer JR, Rosenberg L. Obesity and weight gain in relation to incidence of sarcoidosis in US black women: data from the Black Women's Health Study. Chest. 2015;147(4):1086-93. https:// doi.org/10.1378/chest.14-1099

12. Moon JS, Ahn SS, Park YB, Lee SK, Lee SW. C-Reactive Protein to Serum Albumin Ratio is an independent predictor of all-cause mortality in patients with ANCA-Associated vasculitis. Yonsei Med J. 2018;59(7):865-71. https://doi.org/10.3349/ ymj.2018.59.7.865

13. Gvozdenovic BS, Mihailovic-Vucinic V, Vukovic M, Lower EE, Baughman RP, Dudvarski-llic A, et al. Effect of obesity on patient-reported outcomes in sarcoidosis. Int J Tuberc Lung Dis. 2013;17(4):559-64. https://doi.org/10.5588/ijtld.12.0665

14. Drent M, Crouser ED, Grunewald J. Challenges of sarcoidosis and its management. N Engl J Med. 2021;385(11):1018-32. https://doi.org/10.1056/NEJMra2101555 